\title{
TITLE:
}

\section{ES and iPS cell research for cardiovascular regeneration.}

$\operatorname{AUTHOR}(S)$ :

Yamashita, Jun K

\section{CITATION:}

Yamashita, Jun K. ES and iPS cell research for cardiovascular

regeneration.. Experimental cell research 2010, 316(16): 2555-2559

ISSUE DATE:

2010-10-01

URL:

http://hdl.handle.net/2433/126734

\section{RIGHT:}

C 2010 Elsevier Inc.; この論文は出版社版でありません。引用の際には 出版社版をご確認ご利用ください。; This is not the published version. Please cite only the published version. 


\section{ES and iPS cell research for cardiovascular regeneration}

Jun K. Yamashita ${ }^{1,2}$

${ }^{1}$ Center for iPS Cell Research and Application, Institute for Integrated

Cell-Material Sciences, Kyoto University, Japan

${ }^{2}$ Laboratory of Stem Cell Differentiation, Stem Cell Research Center, Institute for Frontier Medical Sciences, Kyoto University, Japan

Keywords:

Induced pluripotent stem cells, heart, blood vessel, regeneration, drug discovery

Correspondence to:

Jun K. Yamashita, M.D., Ph.D.

Laboratory of Stem Cell Differentiation, Stem Cell Research Center,

Institute for Frontier Medical Sciences, Kyoto University

53 Shogoin Kawahara-cho, Sakyo-ku, Kyoto 606-8507 Japan

TEL: +81-75-751-3853, FAX: +81-75-751-4824

e-mail: juny@frontier.kyoto-u.ac.jp

URL: http://www.frontier.kyoto-u.ac.jp/es02/index.htm 


\section{Abstract}

Embryonic stem (ES) cells and induced pluripotent stem (iPS) cells, which are ES-like stem cells induced from adult tissues, are twin stem cells with currently (with the exception of fertilized eggs) the broadest differentiation potentials. These two stem cells show various similarities in appearance, maintenance methods, growth and differentiation potentials, i.e. theoretically, those cells can give rise to all kinds of cells including germ-line cells. Generation of human ES and iPS cells is further facilitating the researches towards the realization of regenerative medicine. The following three issues are important purposes of ES and iPS cell researches for regenerative medicine: 1) Dissection of differentiation mechanisms, 2) Application to cell transplantation, and 3) Drug discovery. In this review, the current status of cardiovascular regenerative trials using ES and iPS cells is briefly discussed. 


\section{Introduction}

Cardiovascular diseases are the major causes of death in industrialized countries. Cardiovascular cells are, therefore, one of the most important targets in regenerative medicine. The regeneration of cardiomyocytes, which principally cannot proliferate and regenerate in the adult, is particularly expected to bring new hopes to cure cardiac diseases. Many cell types, such as endothelial progenitor cells from blood or bone marrow, cardiac progenitor cells from the heart, mesenchymal cells from bone marrow or other tissues, and ES and iPS cells, are currently being examined as cell sources for cardiovascular regenerative cell therapy [1]. Previously, the authors established a novel ES and iPS cell differentiation system, which can reproduce the early cardiovascular development processes in vitro [2,3,4]. Using and expanding this system as a tool for differentiation studies, cell transplantation and drug discovery, we are trying to explore novel cardiovascular regenerative strategies.

Differentiation of cardiovascular cells from ES and iPS cells

1) Differentiation strategies - embryoid bodies vs. stepwise methods -

To induce ES (or iPS) cell differentiation, embryoid bodies (EBs) which form as aggregates of ES cells are often used. Spontaneous differentiation of 
ES cells occurs in EBs by the interaction of cells within EBs, locally mimicking the body plan in the embryo. EBs contain various cell types including cardiomyocytes, endothelial cells (ECs) and mural cells (MCs; pericytes in capillary vessels and vascular smooth muscle cells in arteries and veins) and often form blood vessel-like structures [5]. Though the EB method is convenient for inducing differentiation and should be suitable for a large-scale preparation of cell sources, the method possesses several weak-points in dissecting cellular and molecular mechanisms during differentiation such as: i) Difficulty to dissect the differentiation mechanisms by highlighting cells and signals of interest in EBs, ii) Difficulty to directly observe differentiating cells at the cellular level by microscopy, and iii) Difficulty to conduct single cell analysis of differentiation.

To overcome these disadvantages in EB cultures, the authors developed a 2-dimentional culture-based, stepwise cardiovascular differentiation system (Figure) $[2,3,6]$. In this system, Flk $1^{+}$mesoderm cells are first induced from undifferentiated ES cells in a monolayer culture of ES cells in the absence of LIF (leukemia inhibitory factor). Flk $1^{+}$cells, presumptive lateral plate mesoderm, are purified by FACS (flow cytometry-assisted cell sorting) using anti-Flk1 antibody. Various cardiovascular cells are then induced by re-culture of purified Flk $1^{+}$cells as common precursor cells. Though this system requires purification and re-culture processes, it is amenable for easy monitoring and 
analysis of differentiating cells at the cellular level. To dissect molecular and cellular mechanisms of cell differentiation or to apply ES/iPS cell differentiation system to screen small molecules, such stepwise and systematic methods are very powerful (see below).

2) Differentiation of vascular cells - vasculodiversity and a constructive approach -

Blood vessels consist of two cell types, ECs and MCs. The majority of vascular cells are considered to be derived from the mesoderm. Expression of Flk1 (also designated as vascular endothelial growth factor receptor-2 (VEGF-R2)) is an indicator of the lateral plate mesoderm [7]. Flk1 is also the earliest functional differentiation marker for blood and ECs [8]. The Flk1 ligand, VEGF, is a key factor for EC differentiation. VEGF-A heterozygotes die early in gestation due to failure in vascular system formation, indicating that strict regulation of VEGF function is critical in normal vascular formation [9]. Recent reports for various molecular markers and functional molecules for arterial, venous, and lymphatic ECs [10] provide a novel research field as "vasculodiversity". A transmembrane ligand ephrinB2 and its receptor tyrosine kinase EphB4 are the first reported markers for arterial and venous ECs, respectively. The ephrinB2-EphB4 system is essential to establish mature blood vessel system with arterial-venous identity. Notch (Notch 1, 4) and its cell-surface ligands 
(Delta like-1 [Dll1], Dll4, and Jagged1 and 2) are expressed in arteries but not in veins. Genetic studies of Notch signaling components have shown that Notch signaling is essential for proper formation of the developing vasculature and arterial specification. On the other hand, insufficient Notch activation during angioblast differentiation to ECs leads ECs to venous fate. COUP-TFII, an orphan nuclear receptor transcription factor, was reported to repress Notch signaling through suppressing neuropilin1 expression to maintain vein identity. Lymphatic ECs originate from venous ECs. A subset of venous ECs expressing LYVE1, which are competent to lymphatic differentiation, are committed into lymphatic ECs with the expression of Prox1 homeobox transcription factor. Prox 1 is considered as the most specific and functional lymphatic EC marker. The author's group succeeded in inducing a variety of vascular cells from mouse ES cells using the stepwise ES cell differentiation system [2,5,11]. ECs and MCs are specifically induced from ES cell-derived Flk1+ cells when they are cultured with VEGF and serum. In this condition, induced ECs mainly show venous phenotype. When cyclic AMP (cAMP) signaling is simultaneously stimulated with VEGF, arterial ECs are induced. A multifunctional polypeptide, adrenomedullin (AM), which exerts its function by increasing the levels of intracellular cAMP, is a candidate endogenous ligand that activates cAMP [12]. Prox1-positive lymphatic ECs are induced [13] when Flk1+ cells are cultured on OP9 cells [14], which are stroma cells established from bone marrow of op/op 
(monocyte colony stimulating factor deficient) mice. Lymphatic ECs are also induced with EB methods. The authors group succeeded in inducing all three EC phenotypes, arterial, venous, and lymphatic ECs from ES cells [10].

Recently, novel roles of cAMP signaling in EC differentiation and arterial specification were demonstrated with the stepwise method. That is, a cAMP downstream gene, protein kinase A (PKA), specifically upregulates selective and sensitive receptors for $\mathrm{VEGF}_{165}$, Flk1 and Neuropilin1, in vascular progenitors, and enhances the "sensitivity of the progenitors" to VEGF 165 by more than 10 times [15]. PKA activation increased the total EC number that appeared from Flk $1^{+}$cells, but had no effect on arterial-venous specification. Arterial specification was caused by another pathway activated by cAMP, that is, Notch and GSK3 $\beta$-mediated $\beta$-catenin signaling [16]. Notch and $\beta$-catenin signaling, both of which are activated through phosphatidylinositol-3 kinase downstream of cAMP, converges into single protein complex on arterial genes. The effect of cAMP in arterial specification was completely reproduced with neither Notch nor $\beta$-catenin, but with simultaneous activation. Thus, two distinct roles of cAMP pathways, common EC differentiation and arterial EC specification, were demonstrated though a constructive approach by building up each molecular functions to reproduce cell differentiation process in vitro $[5,10]$. The stepwise and constructive reproduction of vascular developmental processes with ES cell differentiation system can provide novel understanding in cellular and 
molecular mechanisms of vascular development from a new point of view.

3) Differentiation of cardiomyocytes, progenitors or stem cells

Cardiomyocytes are principally mesoderm derivatives. Mesodermal cells give rise to two cardiac progenitor populations that exist in so-called, primary heart field and secondary heart field $[1,17,18]$. Primary heart field is derived from the anterior splanchnic mesoderm and form cardiac crescent. Primary heart field is positive for Nkx2.5, Tbx5, and Hand1, and gives rise to mainly the left ventricle. Secondary heart field originates from the pharyngeal mesoderm and is situated medially to the primary heart field. Secondary heart field is positive for isl1, Tbx1, FGF8 and 10, and gives rise to mainly the right ventricle, outflow tract and inflow region. In addition to these two populations, proepicardial cells and neural crest cells also contribute to the heart structure [1].

Cardiomyocytes are one of the first cell types induced from ES cells. Appearance of self-beating cells in EBs was reported four years after the derivation of mouse ES cells [19]. The stepwise method showed that ES cell-derived Flk1 ${ }^{+}$cells can give rise to vascular cells as well as cardiomyocytes [3]. When Flk1+ cells were cultured on OP9 stroma cells, self-beating cardiomyocytes appeared in 4-5 days. ES cell-derived cardiac progenitors at a single cell level were first reported as $\mathrm{Flk} 1^{+} / \mathrm{CXCR} 4^{+} /$vascular endothelial 
cadherin- (FCV) cells [3]. Flk1, Nkx2.5, and/or islet1 were reported to mark multipotent cardiac progenitor population [20]. Though these markers mainly represent lateral plate mesoderm, primary heart field, and secondary heart field, respectively, these marker expressions overlap each other [21]. For example, islet1 is also expressed in $\mathrm{Flk} 1^{+}$mesoderm, and $\mathrm{Nkx} 2.5$ is expressed in both heart fields. These progenitor populations should therefore be partially overlapping, and the relationship among them should be further clarified. In human ES cells, KDR (human Flk1)+ cells and Isl1+ cells were independently reported to be multipotent cardiovascular progenitors [22,23]. As for cardiac stem cells, some reports show clonal potential of tissue-derived cardiac stem cells, such as c-kit ${ }^{+}$cells or cardiac side population cells [24]. Though expansion of ES cell-derived progenitor cells was reported with a sphere [25] or feeder cell methods [23], the establishment of ES cell-derived cardiac stem cells has not been completely demonstrated.

Cardiomyocyte induction from mouse iPS cells were first reported in 2008 [4,26,27]. Cardiomyocytes could be induced from mouse iPS cells with similar methods from mouse ES cells with EBs or stepwise methods. Various cardiovascular cells, cardiomyocytes, arterial, venous, and lymphatic ECs, and blood cells, were systematically induced from Flk $1^{+}$progenitor cells [4]. Comparable levels of cardiovascular cells could be induced from iPS cells and ES cells. As for human iPS cells, cardiomyocyte induction using EB methods was reported 
for the first time in 2009 [28]. Though functional analyses of induced cardiomyocytes suggest that human cardiac cell models could be established from human iPS cells, induction efficiency and stability are still not sufficient, especially for cell therapy purpose. Further improvements for more robust induction methods are still required.

Application to cell transplantation

As establishment of human iPS cells from human tissues can avoid the legal and ethical controversy over human ES cells, iPS cells are now one of the most promising cell sources for cardiac regenerative cell therapy. Nevertheless, many hurdles have yet to be overcome before the realization of cardiac regeneration by iPS cells.

There are various cardiovascular lineage cells. What cells are suitable for cardiac regeneration? Cardiomyocytes? Cardiac progenitors? More specific cardiac cells such as pacemaker cells? Vascular cells? Mesenchymal cells? Alternatively, a mixture of different cell types? Many studies are now ongoing all over the world. A large-scale preparation and injection of human ES cell-derived cardiomyocytes $\left(10^{7}\right.$ cells) are reported to be able to ameliorate cardiac function [29]. FCV cardiac progenitor cells from mouse ES cells were 
shown to efficiently generate cardiomyocytes after cell transplantation [21]. Recently, importance of non-cardiomyocytes in cardiac regeneration is highlighted as a source of various humoral factors that help cardiac regeneration in a paracrine fashion [30]. Transplantation of cardiomyocyte/non-cardiomyocyte mixtures may be a good strategy for efficient regeneration. Novel transplantation technologies such as cardiosphere (cardiac cell aggregates)[31] and cardiac cell sheets using temperature-responsive culture surface [32], would support mixture transplantation strategies. Cardiovascular progenitors, which can efficiently give rise to cardiomyocytes as well as endothelial cells and mural cells, should be a good cell source for mixture strategies. In addition to such technical hurdles, elimination of undifferentiated ES/iPS cells to avoid teratoma formation is, by far the most critical issue for the safety of ES/iPS cell therapy. Recently, an iPS cell-specific feature in the teratoma-forming propensity was reported, where some iPS cell lines showed a "differentiation-resistant" phenotype [33]. In a particular iPS cell lines, which may be incompletely reprogrammed, undifferentiated cells persist even after induction of differentiation resulting in teratoma formation after transplantation. Establishment of standard for safe iPS cells would be critical to develop iPS cell therapy. 


\section{Drug discovery}

iPS cell technology, which enables the establishment of patient-specific pluripotent stem cells and patient cell models, brings two new hopes in drug discovery. One is in vitro screening for adverse or toxic effects of drugs. The other is discovering new drugs for currently incurable diseases.

\section{1) Safety test}

Establishment of cardiac cell models from human iPS cells offers novel tools for drug safety test. QT elongation is a critical adverse effect caused by inhibition of human ERG (HERG) ion channel. Currently, so-called HERG test, in which inhibitory effects of chemical substances are evaluated with HERG-overexpressed cell lines (such as HEK293), is mainly used for safety screening of QT elongation. HERG test often shows false negative results, that is, though HERG test is negative, QT elongation occurs in patients (ex. dl-sotarol). When inhibitory effects of substances on HERG current were evaluated using human cardiomyocytes prepared from human ES/iPS cells in vitro, the in vitro results precisely reflected in vivo QT elongation [34]. Human cell models, thus, are potent tool for drug safety test which may drastically simplify and facilitate drug development.

2) Cardiac regenerative drugs

It would be ideal if cardiac regeneration could be achieved with drugs. 
Some trials to discover small molecules which promote cardiomyocyte differentiation are being performed using ES cell differentiation systems. Cardiogenol, ascorbic acid, isoxazolyl-serines, sulfonyl hydrazones, and so on are reported to enhance cardiomyocyte differentiation from ES cells using EB methods [35]. Nevertheless, as these substances were added to EBs (or P19 carcinoma cell lines) from the initial step of differentiation, target cells or processes to which these substances act on are unclear. For example, cardiogenol and sulfonyl hydrazones should act on mesoderm induction stage. Differentiation-stage specific screening and evaluation using stepwise differentiation methods would be more powerful and suitable to discover cardiac regenerative drugs. Indeed, the authors recently demonstrated that an immunosuppressant, cyclosporin-A (CSA), showed a novel effect specifically acting on mesoderm cells to drastically increase cardiac progenitors (FCV cells) as well as cardiomyocytes by 10-20 times [21]. CSA had an effect on specific induction of cardiac lineage from mesoderm, that is, on cardiac commitment process. Drugs acting on the late stages of cardiac differentiation, such as cardiac commitment, differentiation, and cardiomyocyte proliferation, should be promising targets as cardiac regenerative drugs.

Novel perspective - Epigenetic memory and iCM - 
Currently, iPS cells can be established from various cell types. Recent studies suggest that differentiation properties of iPS cells should be affected by their origin (personal communication). iPS cells established from blood cells have a tendency to well differentiate to blood cells but not to other lineages. This phenomenon suggests that epigenetic information as blood cells are still persistent in some degree as a kind of cell memory even after iPS cell derivation process, though the molecular identity of the epigenetic memory is still unknown. If iPS cells could be induced from cardiomyocytes, those iPS cells may be ideal for the efficient preparation of cardiomyocytes.

Recently, direct conversion of fibroblasts to functional neurons (iN cells) was reported by transduction of three defined transcription factors [36]. This result indicates that critical combination of transcription factors can induce and reproduce any kind of distinct cell types. Establishment of iN cells instantly prompts direct induction of cardiomyocytes from fibroblasts (iCM). iCM would offer another important option to research, cell therapy, and drug discovery toward cardiovascular regeneration. iPS cell technologies are still expanding, and will continue to bring various new hopes in cardiovascular regeneration. 
References

1. C. Gonzales, T. Pedrazzini, Progenitor cell therapy for heart disease. Exp. Cell Res. 315 (2009) 3077-3085

2. J. Yamashita, H. Itoh, M. Hirashima, M. Ogawa, S. Nishikawa, T. Yurugi, M. Naito, K. Nakao, S.I. Nishikawa, Flk1 positive cells derived from embryonic stem cells serve as vascular progenitors. Nature 408 (2000) 92-96

3. J.K. Yamashita, M. Takano, M. Hiraoka-Kanie, C. Shimazu, P. Yan, K. Yanagi, A. Nakano, E. Inoue, F. Kita, S.I. Nishikawa. Prospective identification of cardiac progenitor potentials by a novel single cell-based cardiomyocyte induction. FASEB J, 19 (2005) 1534-1536

4. G. Narazaki, H. Uosaki, M. Teranishi, K. Okita, B. Kim, S. Matsuoka, S. Yamanaka, J.K. Yamashita, Directed and systematic differentiation of cardiovascular cells from mouse induced pluripotent stem cells. Circulation, 118 (2008) 498-506

5. J.K. Yamashita, Differentiation and diversification of vascular cells from ES cells. Int J Hematol 80 (2004) 1-6

6. S.I. Nishikawa, S. Nishikawa, M. Hirashima, N. Matsuyoshi, H. Kodama, Progressive lineage analysis by cell sorting and culture identifies FLK1+VE-cadherin+ cells at a diverging point of endothelial and hemopoietic lineages. Development.125 (1998) 1747-57 
7. H. Kataoka, N. Takakura, S. Nishikawa, K. Tsuchida, H. Kodama, T.

Kunisada, W. Risau, T. Kita, S.I. Nishikawa, Expressions of PDGF receptor alpha, c-Kit and Flk1 genes clustering in mouse chromosome 5 define distinct subsets of nascent mesodermal cells. Dev Growth Differ. 39 (1997) $729-40$

8. F. Shalaby, J. Ho, W.L. Stanford, K.D. Fischer, A.C. Schuh, L. Schwartz, A. Bernstein, J. Rossant, A requirement for Flk1 in primitive and definitive hematopoiesis and vasculogenesis. Cell 89 (1997) 981-90

9. P. Carmeliet, V. Ferreira, G. Breier, S. Pollefeyt, L. Kieckens, M. Gertsenstein, M. Fahrig, A. Vandenhoeck, K. Harpal, C. Eberhardt, C. Declercq, J. Pawling, L. Moons, D. Collen, W. Risau, A. Nagy, Abnormal blood vessel development and lethality in embryos lacking a single VEGF allele. Nature 380 (1996) 435-439

10. J.K. Yamashita, Differentiation of arterial, venous, and lymphatic endothelial cells from vascular progenitors. Trends Cardiovasc Med 17 (2007) 59-63

11. M. Hirashima, H. Kataoka, S. Nishikawa, N. Matsuyoshi, S. Nishikawa, Maturation of embryonic stem cells into endothelial cells in an in vitro model of vasculogenesis. Blood 93 (1999) 1253-63

12. T. Yurugi-Kobayashi, H. Itoh, T. Schroeder, A. Nakano, G. Narazaki, F. Kita, K. Yanagi, M. Hiraoka-Kanie, E. Inoue, T. Ara, T. Nagasawa, U. Just, K. 
Nakao, S.I. Nishikawa, J.K. Yamashita, Adrenomedullin/cyclic AMP pathway induces Notch activation and differentiation of arterial endothelial cells from vascular progenitors. Arterioscler Thromb Vasc Biol, 26 (2006) $1977-1984$

13. T. Kono, H. Kubo, C. Shimazu, Y. Ueda, M. Takahashi, K. Yanagi, F. Fujita, T. Tsuruo, H. Wada, J.K. Yamashita, Differentiation of lymphatic endothelial cells from embryonic stem cells on OP9 stromal cells. Arterioscler Thromb Vasc Biol, 26 (2006) 2070-2076

14. H. Kodama, M. Nose, S. Niida, S. Nishikawa, S. Nishikawa, Involvement of the c-kit receptor in the adhesion of hematopoietic stem cells to stroma cells. Exp. Hematol. 2 (1992) 979-984

15. K. Yamamizu, K. Kawasaki, S. Katayama, T. Watabe, J.K. Yamashita, Enhancement of vascular progenitor potential by protein kinase A through dual induction of Flk-1 and Neuropilin-1. Blood, 114 (2009) 3707-3716

16. K. Yamamizu, T. Matsunaga, H. Uosaki, H. Fukushima, S. Katayama, M. Hiraoka-Kanie, K. Mitani, J.K. Yamashita, Convergence of Notch and $\beta$-catenin signaling induces arterial fate in vascular progenitors. J Cell Biol, in press

17. F. Rochais, K. Mesbah, R.G. Kelly, Signaling pathways controlling second heart field development, Circ. Res. 104 (2009) 933-942

18. K.R. Chien, I.J. Domian, K.K. Parker, Cardiogenesis and the complex 
biology of regenerative cardiovascular medicine, Science 322 (2008) 1494-1497

19. T.C. Doetschman, H. Eistetter, M. Katz, W. Schmidt, R. Kemler, The in vitro development of blastocyst-derived embryonic stem cell lines: formation of visceral yolk sac, blood islands and myocardium, J Embryol Exp Morphol. $87(1985) 27-45$

20. D.J. Garry, E.N. Olson, A common progenitor at the heart of development. Cell. 127 (2006) 1101-1104

21. P. Yan, A. Nagasawa, H. Uosaki, A. Sugimoto, K. Yamamizu, M. Teranishi, H. Matsuda, S. Matsuoka, T. Ikeda, M. Komeda, R. Sakata, J.K. Yamashita, Cyclosporin-A potently induces highly cardiogenic progenitors from embryonic stem cells. Biochem Biophys Res Commun, 379 (2009) 115-120

22. L. Yang, M.H. Soonpaa, E.D. Adler, T.K. Roepke, S.J. Kattman, M. Kennedy, E. Henckaerts, K. Bonham, G.W. Abbott, R.M. Linden, L.J.Field, G.M. Keller, Human cardiovascular progenitor cells develop from a KDR+ embryonic-stem-cell-derived population, Nature 453 (2008) 524-529

23. L. Bu, X. Jiang, S. Martin-Puig, L. Caron, S, Zhu, Y. Shao, D.J. Roberts,P.L. Huang, I.J. Domian, K.R. Chien, Human ISL1 heart progenitors generate diverse multipotent cardiovascular cell lineages, Nature 460 (2009) 113-118

24. L. Barile, E. Messina, A. Giacomello, E. Marban, Endogenous cardiac stem cells, Prog.Cardiovasc.Dis. 50 (2007) 31-48 
25. K. Tateishi, E. Ashihara, S. Honsho, M. Takehara, T. Nomura, T. Takahashi, T. Ueyama, M. Yamagishi, H. Yaku, H. Matsubara, H. Oh, Human cardiac stem cells exhibit mesenchymal features and are maintained through Akt/GSK-3ß signaling, Biochem Biophys Res Commun 352 (2007) 635-641

26. C. Mauritz, K. Schwanke, M. Reppel, S. Neef, K. Katsirntaki, L.S. Maier, F. Nguemo, S. Menke, M. Haustein, J. Hescheler, G. Hasenfuss, U. Martin, Generation of functional murine cardiac myocytes from induced pluripotent stem cells. Circulation. 118 (2008) 507-517

27. K. Schenke-Layland, K.E. Rhodes, E. Angelis, Y. Butylkova, S. Heydarkhan-Hagvall, C. Gekas, R. Zhang, J.I. Goldhaber, H.K. Mikkola, K. Plath, W.R. MacLellan, Reprogrammed mouse fibroblasts differentiate into cells of the cardiovascular and hematopoietic lineage, Stem Cells. 26 (2008) $1537-1546$

28. J. Zhang, G.F. Wilson, A.G. Soerens, C.H. Koonce, J. Yu, S.P. Palecek, J.A. Thomson, T.J. Kamp, Functional cardiomyocytes derived from human induced pluripotent stem cells, Circ Res. 104 (2009) e30-41

29. I. Kehat, L. Khimovich, O. Caspi, A. Gepstein, R. Shofti, G. Arbel, I. Huber, J. Satin, J. Itskovitz-Eldor, L. Gepstein, Electromechanical integration of cardiomyocytes derived from human embryonic stem cells. Nat Biotechnol. $22(2004) 1282-1289$

30. M. Gnecchi, Z. Zhang, V.J. Dzau, Paracrine mechanisms in adult stem cell 
signaling and therapy, Circ. Res. 103 (2008) 1204-1219

31. P.V. Johnston, T. Sasano, K. Mills, R. Evers, S.T. Lee, R.R.Smith, A.C. Lardo, S. Lai, C. Steenbergen, G. Gerstenblith, R. Lange, E. Marban, Engraftment, differentiation, and functional benefits of autologous cardiosphere-derived cells in porcine ischemic cardiomyopathy. Circulation 120 (2009) 1075-1083

32. T. Shimizu, H.Sekine, M. Yamato, T. Okano, Cell sheet-based myocardial tissue engineering: new hope for damaged heart rescue. Curr. Pharm. Des. 15 (2009) 2807-2814

33. K. Miura, Y. Okada, T. Aoi, A. Okada, K. Takahashi, K. Okita, M. Nakagawa, M. Koyanagi, K. Tanabe, M. Ohnuki, D. Ogawa, E. Ikeda, H. Okano, S. Yamanaka, Variation in the safety of induced pluripotent stem cell lines. Nat Biotechnol. 27 (2009) 743-745

34. Y. Asai, M. Tada, T.G. Otsuji, N. Nakatsuji, Combination of functional cardiomyocytes derived from human stem cells and a highly-efficient microelectrode array system: an ideal hybrid model assay for drug development. Curr. Stem Cell Res. Ther. (2010) Epub ahead of print

35. E. Willems, P.J. Bushway, M. Mercola, Natural and synthetic regulators of embryonic stem cell cardiogenesis. Pediatr Cardiol. 30 (2009) 635-642

36. T. Vierbuchen, A. Ostermeier, Z.P. Pang, Y. Kokubu, T.C. Sudhof, M. Wernig, Direct conversion of fibroblasts to functional neurons by defined factors. Nature 463 (2010) 1035-1042 
Figure: Systemic and stepwise cardiovascular cell differentiation system with mouse ES/iPS cells 
Undifferentiated ES \& iPS cells

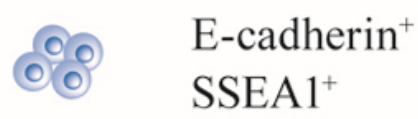

\section{Mesoderm}
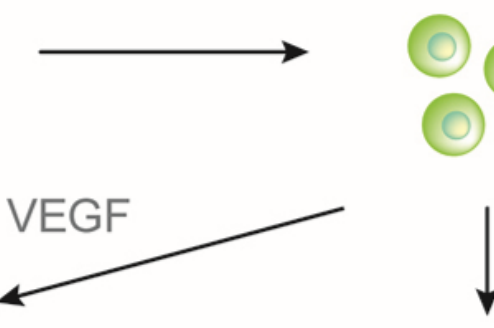

Flk1 ${ }^{+}$

E-cadherin

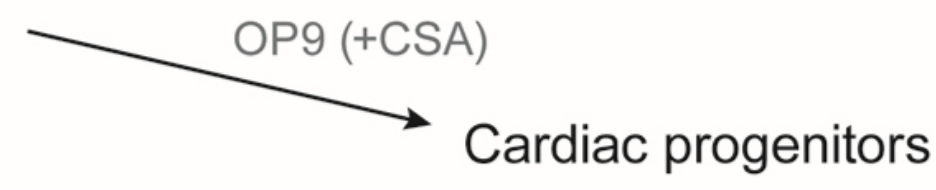

Flk1

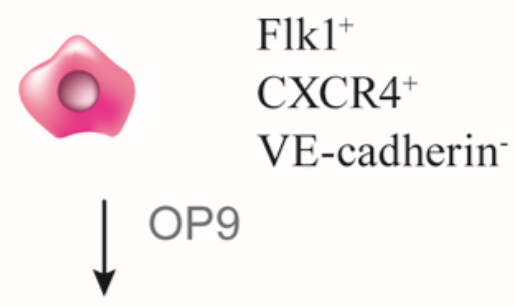

\section{Blood cells}

Endthelial cells

Mural cells

Cardiomyocytes
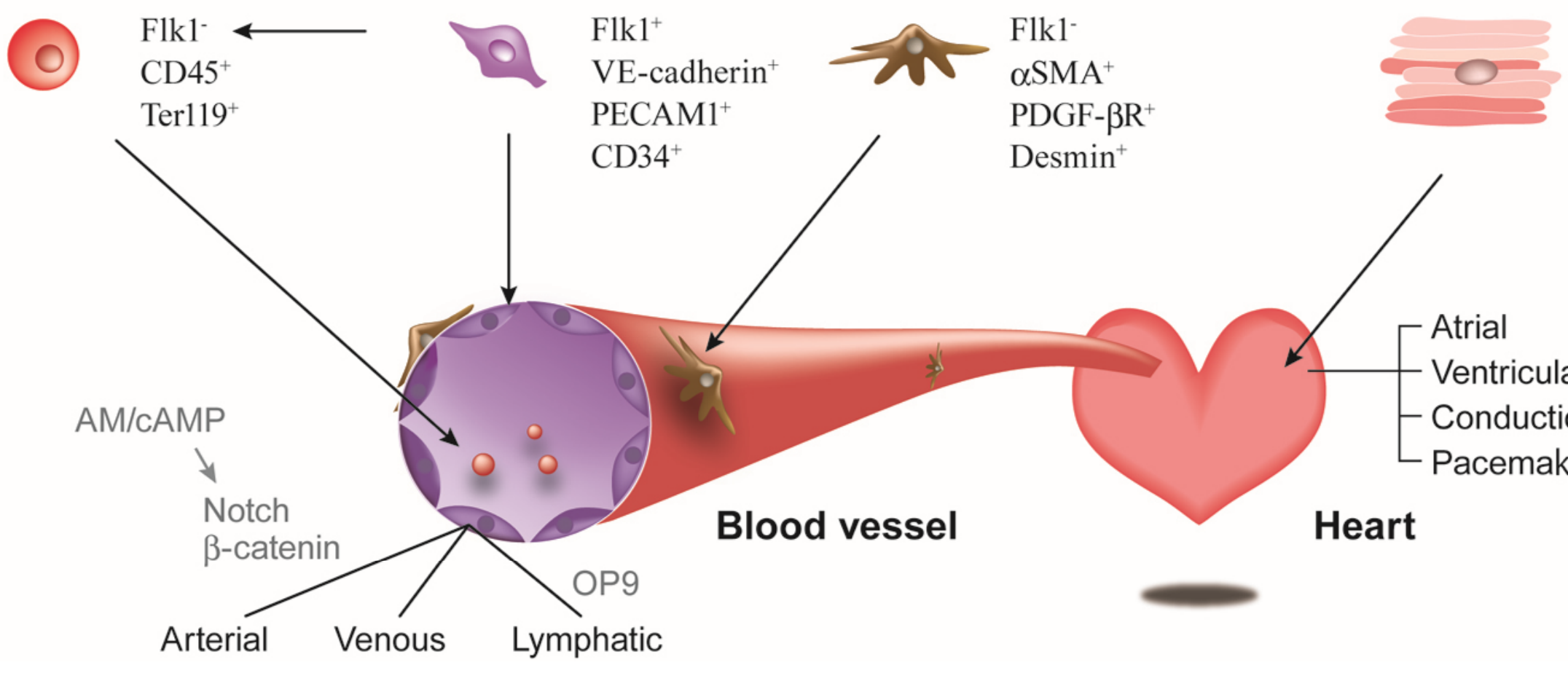

Flk1' $\alpha \mathrm{MHC}^{+}$ $\mathrm{ANP}^{+}$ GATA- $4^{+}$ $\mathrm{Nkx} 2.5^{+}$ 